\title{
新型酰基硫腿及酰基艮的合成及其杀虫活性
}

\author{
徐志红 ${ }^{a, b}$ 刘 斌 ${ }^{a}$ 董宏波 ${ }^{a}$ 王明安*,a \\ ( ${ }^{a}$ 中国农业大学应用化学系 北京 100193) \\ $\left({ }^{b}\right.$ 长江大学农学院 荆州 434205)
}

\begin{abstract}
摘要 以 5-(4-氨基苄基)-2-硫代-2,4-咪唑啉二酮、5-(4-氨基芐基)-2,4-咪唑啉二酮和 5-(4-氨基苯基)-2,4-咪唑啉二酮为原 料, 分别与取代苯/吡啶甲酰基异氧酸/异硫氰酸酯反应合成了 15 个新型含 2,4-咪唑啉二酮杂环的酰基硫脲及酰基脲类 化合物. 它们的结构经 IR, ${ }^{1} \mathrm{H}$ NMR 和 HR-ESI-MS 的确证. 以粘虫(Mythimna separata)、棉铃虫(Helicoverpa armigera), 玉米蛽(Ostrinia nubilalis)、小菜蛾(Plutella xylostella)、蚜虫(Aphis laburni) 和蚊幼虫(Culex pipiens pallens) 为试虫测试了 它们的生物活性，结果表明部分化合物特别是 5-(4-氨基苯基)-2,4-咪唑啉二酮-2,4,6-三氯苯甲酰基硫脲(4)在 $600 \mathrm{mg} / \mathrm{L}$ 测试浓度下对棉铃虫、玉米螟、小菜蛾的死亡率分别为 $85 \%, 90 \%$ 和 $100 \%$, 在 10 和 $5 \mathrm{mg} / \mathrm{L}$ 测试浓度下对蚊幼虫的死 亡率均为 $100 \%$, 表现出良好的杀虫活性.
\end{abstract}

关键词 5-(4-氨基苯基/芐基)-2,4-咪唑啉二酮; 酰基硫脲; 酰基脲; 合成; 杀虫活性

\section{Synthesis, Insecticidal Activity of New Benzoylthioureas and Benzoylureas}

\author{
Xu, Zhihong ${ }^{a, b} \quad$ Liu, Bin ${ }^{a} \quad$ Dong, Hongbo ${ }^{a} \quad$ Wang, Mingan ${ }^{*, a}$ \\ ( ${ }^{a}$ Department of Applied Chemistry, China Agricultural University, Beijing 100193) \\ $\left({ }^{b}\right.$ College of Agriculture, Yangtze University, Jingzhou 434205)
}

\begin{abstract}
Fifteen novel benzoylthioureas and benzoylureas containg hydantoin ring were synthesized by the reaction of 5-(4-aminophenyl)- and 5-(4-aminobenzyl)-hydantoin or 5-(4-aminobenzyl)-thiohydantoin with substituted benzoyl or pyridineacyl isocyanates and isothiocyanates, respectively. Their structures were characterized by the IR, ${ }^{1} \mathrm{H}$ NMR and HR-ESI-MS spectral data. Their bioactivities were evaluated with the insects Mythimna separata, Helicoverpa armigera, Ostrinia nubilalis, Plutella xylostella, Aphis laburni and Culex pipiens pallens. The results showed that 5-(4-aminophenyl)hydantoin-2,4,6trichlorobenzoylthiourea (4) exhibit $85 \%, 90 \%$ and $100 \%$ mortality against $H$. armigera, O. nubilalis and P.xylostella at the concentration of $600 \mathrm{mg} / \mathrm{L}, 100 \%$ mortality against larva of $C$. pipiens at the concentration of $5 \mathrm{and} 10 \mathrm{mg} / \mathrm{L}$, respectively. Keywords 5-(4-aminophenyl/benzyl)-hydantoin; benzoylthiourea; benzoylurea; synthesis; insecticidal activity
\end{abstract}

酰基硫艮及酰基脲因表现出良好的杀虫、杀菌、除 草、抗病毒等生物活性，近年来它们成为倍受关注的一 类化合物，特别是酰基脲类化合物中有数个如除虫脲、 定虫隆、氟幼脲、氟铃脲、氟虫隆等已经开发为商品化 的杀虫剂, 更加吸引人们的研究兴趣 ${ }^{[1 \sim 8]}$. 在酰基硫脲 及酰基脲的结构优化研究中, 对酰基进行的大量结构改 造结果证明, 苯甲酰基的苯环上引入氯、氟原子的化合 物具有优异的杀虫活性, 而在对芳胺的结构改造中将各 种取代基、杂环、肜醚、二芳醚结构等引入到苯环上以
及替换为杂环芳胺等都发现了大量高活性的化合 物 $^{[3 \sim 5]}$, 但是迄今未见他人将 2,4 -咪唑啉二酮杂环引入 到苯环上合成酰基硫脲及酰基嫝的报道. 本课题组在研 究含 2,4-咪唑啉二酮杂环化合物中, 发现合成的一些磷 酰胺和磷酰胺酯类化合物表现出较好的杀虫活性 ${ }^{[9,10]}$, 因此本文作者根据活性亚结构拼接原理希望将 2,4-咪唑 啉二酮活性结构片段引入到具有优异杀虫、杀菌、除草 等活性的酰基硫脲及酰基脲结构中(Scheme 1)，以进一 步观测 2,4-咪唑啉二酮杂环对酰基硫脲及酰基艮类化合

\footnotetext{
*E-mail: wangma@cau.edu.cn

Received July 18, 2014; revised August 11, 2014; published online August 28, 2014

Project supported by the National Key Technologies R\&D Program (No. 2011BAE06B03) and the National Natural Science Foundation of China (No. 20772150).

“十二五” 科技支撑计划(No. 2011BAE06B03)和国家自然科学基金(No. 20772150)资助项目.
} 
物生物活性的影响, 在前文中我们已经报道了一类新的 含 2,4-咪唑啉二酮杂环酰基硫脲的合成及生物活性, 发 现部分化合物表现出优异的除草活性, 但是整体化合物 杀菌活性很差 ${ }^{[11]}$, 本文继续报道含 2,4-咪唑啉二酮杂环 的酰基硫脲和酰基腿类化合物的合成(Scheme 2)以及它 们的杀虫活性 ${ }^{[1 \sim 8]}$.

\section{1 结果与讨论}

\section{1 目标化合物的合成及谱学特征}

中间体胺的合成中最后一步硝基采用 $\mathrm{Pd} / \mathrm{C}$ 催化氢 化还原, 后处理简单收率高, 显著优于用氯化铵和还原 铁粉在回流条件下的实验结果 ${ }^{[10,11]}$. 目标化合物酰基硫 脲的合成主要在酰基异硫氰酸酯的制备, 通过加入聚乙 二醇-400, 提高了反应转化率, 最后生成的酰基硫脲纯 度高易于通过重结晶进一步纯化, 而在酰基艮的合成中 关键要使用新制备的 2,6-二氟苯甲酰异氧酸酯, 在后处
理过程中由于溶解度问题，经石油醚/乙酸乙酯 $/ N, N-$ 二 甲基甲酰胺(DMF)体系重结晶只能得到部分高纯度样 品, 母液需经石油醚/乙酸乙酯/甲醇进一步处理才能获 得大量样品，否则收率非常低. 尽管文献报道了一些合 成酰基脲的新方法如单取代脲与卤代苯及一氧化碳在 钯试剂催化下的 $N$-酰基化反应 ${ }^{[12 \sim 14]}$, 但是由于试剂昂 贵，条件苛刻，本文仍采用经典的方法来完成.

目标化合物酰基硫脲的谱学特征与前文 ${ }^{[11]}$ 一致, 本文不再讨论. 酰基脲的红外光谱在 3121 3224 和 1783 1668 $\mathrm{cm}^{-1}$ 范围可以分别观测到活泼质子和羰基 的特征吸收峰, 在 ${ }^{1} \mathrm{H}$ NMR 谱中在 $\delta$ 11.46 7.93 范围 内可以观测到 4 个活泼质子的特征吸收峰，如化合物 14 的 4 个活泼质子吸收峰化学位移分别在 $\delta 11.44,10.79$, 10.21 和 8.39, 在酰基硫腿和酰基脲的高分辨质谱中，都 能观测到 $[\mathrm{M}+\mathrm{H}]^{+}$或 $[\mathrm{M}+\mathrm{Na}]^{+}$的准分子离子，这些结果 为结构分析提供了有力的证据.

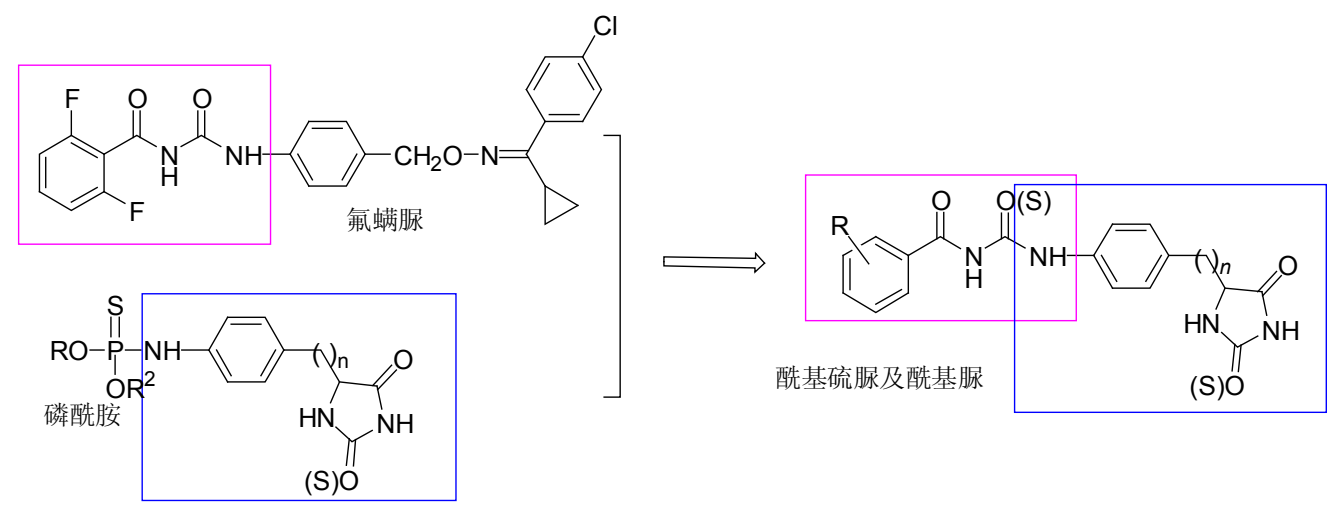

Scheme 1
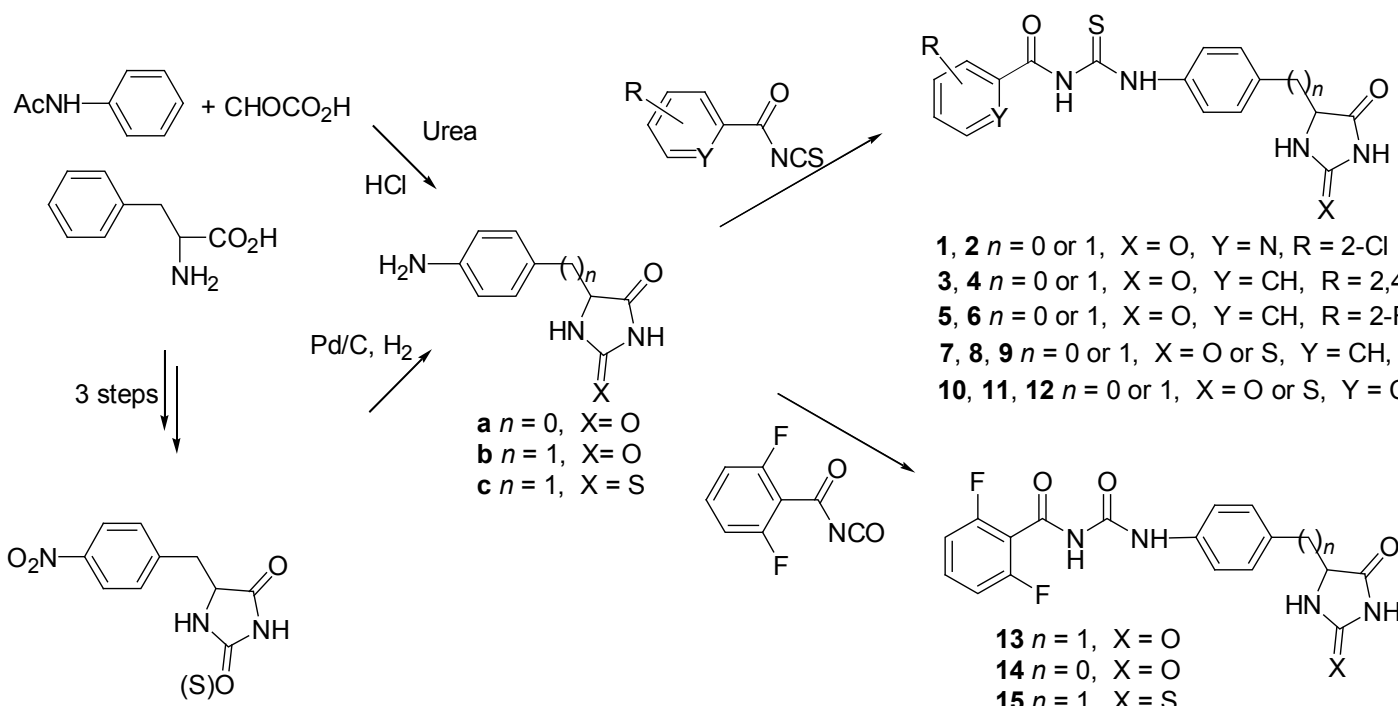

$1,2 n=0$ or $1, \mathrm{X}=\mathrm{O}, \mathrm{Y}=\mathrm{N}, \mathrm{R}=2-\mathrm{Cl}$

$3,4 n=0$ or $1, \mathrm{X}=\mathrm{O}, \mathrm{Y}=\mathrm{CH}, \mathrm{R}=2,4,6-\mathrm{Cl}_{3}$

$5,6 n=0$ or $1, X=O, Y=C H, R=2-\mathrm{F}$

7, 8, $9 n=0$ or $1, \mathrm{X}=\mathrm{O}$ or $\mathrm{S}, \mathrm{Y}=\mathrm{CH}, \mathrm{R}=2,6-\mathrm{Cl}_{2}$

$10,11,12 n=0$ or $1, X=O$ or $S, Y=C H, R=2,6-F_{2}$

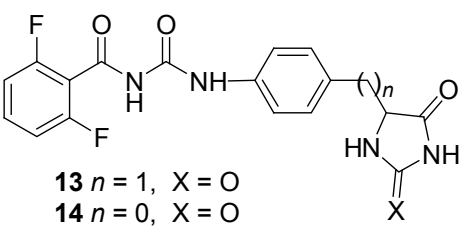

$15 n=1, X=S$

Scheme 2 


\section{2 化合物的杀虫活性}

本文试虫由南开大学元素有机化学国家重点实验 室饲养, 并由南开大学元素有机化学国家重点实验室参 考文献方法 ${ }^{[4,5]}$ 对 15 个酰基硫腿和酰基脲化合物进行了 杀虫活性的测定, 测得的结果如表 1 和表 2 所示. 表 1 的结果说明合成的 15 个酰基硫艮和酰基腿在 $600 \mathrm{mg} / \mathrm{L}$ 时对粘虫(Mythimna separata)、蚜虫(Aphis laburni) 以及 3 个酰基脲对棉铃虫(Helicoverpa armigera) 和玉米蛽 (Ostrinia nubilalis)均没有明显的杀虫活性, 而 12 个酰基 硫腿中化合物 4, 6 9, 11 共 6 个化合物对棉铃虫、玉米 螟和小菜蛾(Plutella xylostella) 显示出良好的杀虫活性, 尤其是 4, 9 和 11 对小菜蛾的致死率分别为 $100 \%, 100 \%$ 和 $86 \%$, 当测试浓度降为 $200 \mathrm{mg} / \mathrm{L}$ 时, 它们仍显示出 $43 \%, 20 \%$ 和 71\%的致死率，这说明在酰基芳环的 2, 6 位 上具有多个氯或氟原子对杀虫活性的提高是至关重要 的. 相对于对照药剂氟螨艮而言, 化合物的致死率明显 降低. 表 2 的结果说明 15 个酰基硫腿和酰基嫝化合物在 $10 \mathrm{mg} / \mathrm{L}$ 浓度时对蚊幼虫(Culex pipiens pallens) 有 12 个 化合物的致死率为 $100 \%$, 特别是化合物 4 在浓度降为 5 和 $2 \mathrm{mg} / \mathrm{L}$ 时, 仍显示出 $100 \%$ 和 40\%的致死率, 而其它 化合物的致死率明显降低, 这说明酰基硫腿化合物 $\mathbf{4}$ 具 有优异的杀蚊幼虫活性, 但在低浓度时仍比氟螨嘱差. 这些结果说明 2,4-咪唑啉二酮杂环的引入没有显著提高 酰基硫脲和酰基艮类化合物的杀虫活性 ${ }^{[15,16]}$. 与文献 [10，11]合成的磷酰胺类化合物相比较, 本文的酰基硫 脲和酰基嘱类化合物对蚜虫没有表现出明显的杀虫活 性, 而 4,9 和 11 对小菜蛾则显示出良好的杀虫活性, 与 磷酰胺类化合物对它们的杀虫活性正好相反, 这可能预
示两类化合物的杀虫作用机制不同.

综合上述实验结果，本文合成的酰基硫艮化合物 $\mathbf{4}$ 对棉铃虫、玉米蛽、小菜蛾和蚊幼虫均表现了良好的杀 虫活性，可以作为先导结构进行进一步的结构优化以提 高它们的杀虫活性.

\section{2 实验部分}

\section{1 仪器与试剂}

Yanagimoto 显微熔点仪(温度计未校正); Bruker DPX $300 \mathrm{MHz}$ 核磁共振仪, TMS 为内标, DMSO- $d_{6}$ 为溶 剂; Brüker Apex II 高分辨质谱仪(ESI-MS). 本实验所用 试剂均为国产或进口分析纯, 无水溶剂用常规方法干燥 处理.

2.2 中间体 5-(4-氨基苯基/苠基)-(2-硫代)-2,4-咪唑啉 二酮 $a, b, c$ 和异硫氭酸酯及异氭酸酯的合成

中间体 $\mathbf{a}, \mathbf{b}$ 和 $\mathbf{c}$ 参照文献 $[10,11]$ 方法合成，硝基还 原一步采用 $\mathrm{Pd} / \mathrm{C}$ 催化氢化还原, 产物的分离在除去催 化剂后脱除溶剂就可以得到化合物，收率分别为 $90 \%$ 和 $92 \%$, 其 m.p., ${ }^{1} \mathrm{H}$ NMR 均与文献 $[10,11]$ 一致.

所有取代苯甲酰异硫氰酸酯参照文献 $[4,5,11]$ 方法 由羧酸通过制备酰氯，再与硫氧酸钾反应合成; $2,6-二$ 氟苯甲酰异氰酸酯参照文献[4，12]方法由 2,6-二氟苯甲 酰胺与草酰氯反应制备, 直接用于下一步反应.

\section{3 目标化合物酰基硫脲及酰基腿的合成}

\subsection{1 酰基硫豚的合成通法}

12 个酰基硫脲化合物 1 12 的合成参照文献[11]方 法合成, 以化合物 3 的合成为例. 在干燥的 $100 \mathrm{~mL}$ 单口

表 1 目标化合物 1 15 的杀虫活性(死亡率/\%)

Table 1 The insecticidal activities (mortality/\%) of title compounds $\mathbf{1} \sim \mathbf{1 5}$

\begin{tabular}{|c|c|c|c|c|c|c|}
\hline Compd. & Conc. $/\left(\mathrm{mg} \cdot \mathrm{L}^{-1}\right)$ & M. separata & H. armigera & O. nubilalis & P. xylostella ${ }^{a}$ & A. laburni \\
\hline 1 & 600 & 10 & 35 & 20 & 0 & 15 \\
\hline 2 & 600 & 6.7 & 15 & 15 & 0 & 19 \\
\hline 3 & 600 & 3.3 & 30 & 35 & 0 & 26 \\
\hline 4 & 600 & 20 & 85 & 90 & $100(43)$ & 21 \\
\hline 5 & 600 & 6.7 & 35 & 30 & 0 & 20 \\
\hline 6 & 600 & 30 & 80 & 75 & 0 & 18 \\
\hline 7 & 600 & 23.3 & 75 & 80 & 0 & 18 \\
\hline 8 & 600 & 36.7 & 70 & 75 & 0 & 21 \\
\hline 9 & 600 & 3.3 & 85 & 85 & $100(20)$ & 28 \\
\hline 10 & 600 & 6.7 & 50 & 40 & 43 & 34 \\
\hline 11 & 600 & 43.3 & 35 & 30 & $86(71)$ & 42 \\
\hline 12 & 600 & 10 & 50 & 55 & 30 & 24 \\
\hline 13 & 600 & 15 & 20 & 25 & - & - \\
\hline 14 & 600 & 10 & 15 & 20 & - & - \\
\hline 15 & 600 & 5 & 10 & 10 & - & - \\
\hline Flucycloxuron & 200 & 100 & 100 & 100 & 100 & 100 \\
\hline
\end{tabular}

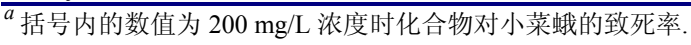


表 2 目标化合物 $1 \sim 15$ 对蚊幼虫(Culex pipiens pallens)的杀 虫活性

Table 2 The insecticidal activities of title compounds $\mathbf{1} \sim \mathbf{1 5}$ against larva of Culex pipiens pallens

\begin{tabular}{cccc}
\hline \multirow{2}{*}{ Compd. } & \multicolumn{3}{c}{ Mortality/\% } \\
\cline { 2 - 4 } & $10 \mathrm{mg} / \mathrm{L}$ & $5 \mathrm{mg} / \mathrm{L}$ & $2 \mathrm{mg} / \mathrm{L}$ \\
\hline $\mathbf{1}$ & 60 & - & - \\
$\mathbf{2}$ & 100 & 30 & - \\
$\mathbf{3}$ & 100 & 30 & - \\
$\mathbf{4}$ & 100 & $\mathbf{1 0 0}$ & 40 \\
$\mathbf{5}$ & 100 & 20 & - \\
$\mathbf{6}$ & 100 & 40 & - \\
$\mathbf{7}$ & 100 & 30 & - \\
$\mathbf{8}$ & 100 & 30 & - \\
$\mathbf{9}$ & 100 & 20 & - \\
$\mathbf{1 0}$ & 100 & 20 & - \\
$\mathbf{1 1}$ & 100 & 20 & - \\
$\mathbf{1 2}$ & 45 & - & - \\
$\mathbf{1 3}$ & 100 & 60 & - \\
$\mathbf{1 4}$ & 100 & 40 & - \\
$\mathbf{1 5}$ & 40 & - & - \\
Flucycloxuron & 100 & 100 & 100 \\
\hline
\end{tabular}

瓶中, 加入 $0.62 \mathrm{~g}$ (3 mmol) 5-(4-氨基苠基)-2,4-咪唑啉 二酩, 用 $25 \mathrm{~mL}$ 乙腈搅拌溶解, 再加入新鲜制备的 2,4,6-三氯苯甲酰基异硫氧酸酯(3 mmol)的 $25 \mathrm{~mL}$ 乙腈 溶液, 室温摚拌过夜, 有白色或黄色沉淀生成. 过滤, 用乙醇/DMF 重结晶或适量乙腈和乙醇洗涤烘干即得产 品, 收率为 $75 \%$. 采用同法制得其它化合物, 它们的结 构表征数据如下.

5-(4-氨基苠基)-2,4-咪唑啉二酮-6-氯吡啶-2-甲酰基 硫艮(1): 白色固体, 收率 86\%. m.p. $226 \sim 228{ }^{\circ} \mathrm{C} ;{ }^{1} \mathrm{H}$ NMR (DMSO- $\left.d_{6}, 300 \mathrm{MHz}\right) \delta: 12.59(\mathrm{~s}, 1 \mathrm{H}), 11.48$ (s, $1 \mathrm{H}), 10.83$ (s, 1H), 8.66 (dd, $J=6.8,2.5 \mathrm{~Hz}, 1 \mathrm{H}), 8.39$ (t, $J=6.8 \mathrm{~Hz}, 1 \mathrm{H}), 7.99$ (s, 1H), $7.96(\mathrm{dd}, J=6.8,2.5 \mathrm{~Hz}$, $1 \mathrm{H}), 7.65$ (d, $J=8.3 \mathrm{~Hz}, 2 \mathrm{H}), 7.24$ (d, $J=8.3 \mathrm{~Hz}, 2 \mathrm{H}), 4.32$ (t, $J=4.8 \mathrm{~Hz}, 1 \mathrm{H}), 2.94(\mathrm{~d}, J=4.8 \mathrm{~Hz}, 2 \mathrm{H})$; IR (KBr) $v$ : 3240, 3056, 1786, 1725, 1682, $1598 \mathrm{~cm}^{-1}$. HR-ESI-MS cacld for $\mathrm{C}_{17} \mathrm{H}_{15} \mathrm{ClN}_{5} \mathrm{O}_{3} \mathrm{~S}[\mathrm{M}+\mathrm{H}]^{+}$404.0584, found 404.0582 .

5-(4-氨基苯基)-2,4-咪唑啉二酮-6-氯吡啶-2-甲酰基 硫脲(2): 白色固体, 收率 89\%. m.p. $213 \sim 215{ }^{\circ} \mathrm{C} ;{ }^{1} \mathrm{H}$ NMR (DMSO- $\left.d_{6}, 300 \mathrm{MHz}\right) \delta: 12.58(\mathrm{~s}, 1 \mathrm{H}), 11.50(\mathrm{~s}$, $1 \mathrm{H}), 10.48$ (s, 1H), 8.66 (dd, $J=6.8,2.5 \mathrm{~Hz}, 1 \mathrm{H}), 8.43$ (s, $1 \mathrm{H}), 8.39$ (t, $J=6.8 \mathrm{~Hz}, 1 \mathrm{H}), 7.97$ (dd, $J=6.8,2.5 \mathrm{~Hz}$, 1H), 7.59 (d, $J=7.4 \mathrm{~Hz}, 2 \mathrm{H}), 7.36$ (d, $J=7.4 \mathrm{~Hz}, 2 \mathrm{H}), 5.20$ (s, 1H); IR (KBr) v: 3220, 3064, 1784, 1721, 1672, 1594 $\mathrm{cm}^{-1}$. HR-ESI-MS cacld for $\mathrm{C}_{16} \mathrm{H}_{13} \mathrm{ClN}_{5} \mathrm{O}_{3} \mathrm{~S}[\mathrm{M}+\mathrm{H}]^{+}$ 390.0428 , found 390.0432 .

5-(4-氨基苠基)-2,4-咪唑啉二酮-2,4,6-三氯苯甲酰
基硫脲(3): 白色固体，收率 75\%. m.p. 204 206 ${ }^{\circ} \mathrm{C} ;{ }^{1} \mathrm{H}$ NMR (DMSO- $\left.d_{6}, 300 \mathrm{MHz}\right) \delta: 12.58(\mathrm{~s}, 1 \mathrm{H}), 11.48(\mathrm{~s}$, 1H), 10.82 (s, 1H), 7.98 (s, 1H), 7.56 (s, 2H), 7.66 (d, J= $8.3 \mathrm{~Hz}, 2 \mathrm{H}), 7.25$ (d, $J=8.3 \mathrm{~Hz}, 2 \mathrm{H}), 4.34(\mathrm{t}, J=4.8 \mathrm{~Hz}$, $1 \mathrm{H}), 2.95$ (d, $J=4.8 \mathrm{~Hz}, 2 \mathrm{H})$; IR (KBr) v: 3244, 3055, $1779,1725,1678,1599 \mathrm{~cm}^{-1}$. HR-ESI-MS cacld for $\mathrm{C}_{18} \mathrm{H}_{14} \mathrm{Cl}_{3} \mathrm{~N}_{4} \mathrm{O}_{3} \mathrm{~S}[\mathrm{M}+\mathrm{H}]^{+}$470.9852, found 470.9854 .

5-(4-氨基苯基)-2,4-咪唑啉二酮-2,4,6-三氯苯甲酰 基硫脲(4): 白色固体，收率 82\%. m.p. 209 210 ${ }^{\circ} \mathrm{C} ;{ }^{1} \mathrm{H}$ NMR (DMSO- $\left.d_{6}, 300 \mathrm{MHz}\right) \delta: 12.59$ (s, 1H), 11.51 (s, 1H), 10.49 (s, 1H), 8.44 (s, 1H), 7.59 (d, $J=7.4 \mathrm{~Hz}, 2 \mathrm{H})$, 7.57 (s, 2H), 7.36 (d, J=7.4 Hz, 2H), 5.20 (s, 1H); IR (KBr) $v: 3234,3065,1780,1726,1677,1596 \mathrm{~cm}^{-1}$. HR-ESI-MS cacld for $\mathrm{C}_{17} \mathrm{H}_{12} \mathrm{Cl}_{3} \mathrm{~N}_{4} \mathrm{O}_{3} \mathrm{~S} \quad[\mathrm{M}+\mathrm{H}]$ 456.9696, found 456.9692 .

5-(4-氨基苠基)-2,4-咪唑啉二酮-2-氟苯甲酰基硫脲 (5): 白色固体, 收率 85\%. m.p. 194 196 ${ }^{\circ} \mathrm{C} ;{ }^{1} \mathrm{H}$ NMR $\left(\mathrm{DMSO}-d_{6}, 300 \mathrm{MHz}\right) \delta: 12.59(\mathrm{~s}, 1 \mathrm{H}), 11.48(\mathrm{~s}, 1 \mathrm{H})$, $10.84(\mathrm{~s}, 1 \mathrm{H}), 8.04 \sim 8.01(\mathrm{~m}, 2 \mathrm{H}), 7.99(\mathrm{~s}, 1 \mathrm{H}), 7.65(\mathrm{~d}$, $J=8.3 \mathrm{~Hz}, 2 \mathrm{H}), 7.45 \sim 7.40(\mathrm{~m}, 2 \mathrm{H}), 7.24(\mathrm{~d}, J=8.3 \mathrm{~Hz}$, $2 \mathrm{H}), 4.35$ (t, $J=4.8 \mathrm{~Hz}, 1 \mathrm{H}), 2.96$ (d, $J=4.8 \mathrm{~Hz}, 2 \mathrm{H})$; IR $(\mathrm{KBr}) v:$ 3248, 3056, 1774, 1728, 1672, $1594 \mathrm{~cm}^{-1}$. HR-ESI-MS cacld for $\mathrm{C}_{18} \mathrm{H}_{16} \mathrm{FN}_{4} \mathrm{O}_{3} \mathrm{~S}[\mathrm{M}+\mathrm{H}]^{+}$387.0927, found 387.0924 .

5-(4-氨基苯基)-2,4-咪唑啉二酮-2-氟苯甲酰基硫脲 (6): 白色固体，收率 81\%. m.p. 201 203 ${ }^{\circ} \mathrm{C}$; ${ }^{1} \mathrm{H}$ NMR $\left(\mathrm{DMSO}-d_{6}, 300 \mathrm{MHz}\right) \delta: 12.57(\mathrm{~s}, 1 \mathrm{H}), 11.50(\mathrm{~s}, 1 \mathrm{H})$, $10.48(\mathrm{~s}, 1 \mathrm{H}), 8.45(\mathrm{~s}, 1 \mathrm{H}), 8.04 \sim 8.00(\mathrm{~m}, 2 \mathrm{H}), 7.58(\mathrm{~d}$, $J=7.4 \mathrm{~Hz}, 2 \mathrm{H}), 7.46 \sim 7.41(\mathrm{~m}, 2 \mathrm{H}), 7.35(\mathrm{~d}, J=7.4 \mathrm{~Hz}$, 2H), 5.21 (s, 1H); IR (KBr) v: 3244, 3060, 1781, 1725, $1675,1598 \mathrm{~cm}^{-1}$. HR-ESI-MS cacld for $\mathrm{C}_{17} \mathrm{H}_{14} \mathrm{FN}_{4} \mathrm{O}_{3} \mathrm{~S}$ $[\mathrm{M}+\mathrm{H}]^{+} \quad 373.0771$, found 373.0774 .

5-(4-氨基苠基)-2,4-咪唑啉二酥-2,6-二氯苯甲酰基 硫脲(7): 白色固体，收率 78\%. m.p. 196 $198{ }^{\circ} \mathrm{C} ;{ }^{1} \mathrm{H}$ NMR (DMSO- $\left.d_{6}, 300 \mathrm{MHz}\right) \delta: 12.58(\mathrm{~s}, 1 \mathrm{H}), 11.47$ (s, 1H), 10.85 (s, 1H), 7.98 (s, 1H), 7.89 (t, J=7.5 Hz, 1H), 7.65 (d, $J=8.3 \mathrm{~Hz}, 2 \mathrm{H}), 7.55$ (d, $J=7.5 \mathrm{~Hz}, 2 \mathrm{H}), 7.24$ (d, $J=8.3 \mathrm{~Hz}, 2 \mathrm{H}), 4.36(\mathrm{t}, J=4.8 \mathrm{~Hz}, 1 \mathrm{H}), 2.98$ (d, $J=4.8$ $\mathrm{Hz}, 2 \mathrm{H})$; IR (KBr) v: 3228, 3059, 1775, 1730, 1674, 1595 $\mathrm{cm}^{-1}$. HR-ESI-MS cacld for $\mathrm{C}_{18} \mathrm{H}_{15} \mathrm{Cl}_{2} \mathrm{~N}_{4} \mathrm{O}_{3} \mathrm{~S}[\mathrm{M}+\mathrm{H}]^{+}$ 437.0242, found 437.0246.

5-(4-氨基苯基)-2,4-咪唑啉二酮-2,6-二氯苯甲酰基 硫脲(8): 白色固体，收率 81\%. m.p. 205 207 ${ }^{\circ} \mathrm{C} ;{ }^{1} \mathrm{H}$ NMR (DMSO- $\left.d_{6}, 300 \mathrm{MHz}\right) \delta: 12.59$ (s, 1H), 11.51 (s, 1H), 10.47 (s, 1H), 8.45 (s, 1H), 7.89 (t, $J=7.5 \mathrm{~Hz}, 1 \mathrm{H})$, 
7.59 (d, $J=7.4 \mathrm{~Hz}, 2 \mathrm{H}), 7.54$ (d, $J=7.5 \mathrm{~Hz}, 2 \mathrm{H}), 7.36$ (d, $J=7.4 \mathrm{~Hz}, 2 \mathrm{H}), 5.22$ (s, 1H); IR (KBr) v: 3241, 3055, 1776, 1728, 1678, $1592 \mathrm{~cm}^{-1}$. HR-ESI-MS cacld for $\mathrm{C}_{17} \mathrm{H}_{13} \mathrm{Cl}_{2} \mathrm{~N}_{4} \mathrm{O}_{3} \mathrm{~S}[\mathrm{M}+\mathrm{H}]^{+}$423.0085, found 423.0079.

5-(4-氨基芐基)-2-硫代-2,4-咪唑啉二酮-2,6-二氯苯 甲酰基硫䏱(9): 微黄色固体, 收率 86\%. m.p. 236 238 ${ }^{\circ} \mathrm{C} ;{ }^{1} \mathrm{H}$ NMR (DMSO- $\left.d_{6}, 300 \mathrm{MHz}\right) \delta: 12.61(\mathrm{~s}, 1 \mathrm{H}), 11.59$ (s, 1H), $11.52(\mathrm{~s}, 1 \mathrm{H}), 10.08(\mathrm{~s}, 1 \mathrm{H}), 7.87(\mathrm{t}, J=7.5 \mathrm{~Hz}$, 1H), 7.65 (d, $J=8.3 \mathrm{~Hz}, 2 \mathrm{H}), 7.55$ (d, $J=7.5 \mathrm{~Hz}, 2 \mathrm{H}), 7.24$ (d, $J=8.3 \mathrm{~Hz}, 2 \mathrm{H}), 4.56$ (t, $J=4.8 \mathrm{~Hz}, 1 \mathrm{H}), 2.98$ (d, $J=4.8$ $\mathrm{Hz}, 2 \mathrm{H}$ ); IR (KBr) v: 3236, 3065, 1778, 1731, 1678, 1598 $\mathrm{cm}^{-1}$. HR-ESI-MS cacld for $\mathrm{C}_{18} \mathrm{H}_{15} \mathrm{Cl}_{2} \mathrm{~N}_{4} \mathrm{O}_{2} \mathrm{~S}_{2}[\mathrm{M}+\mathrm{H}]^{+}$ 453.0013, found 453.0019.

5-(4-氨基苄基)-2,4-咪唑啉二酮-2,6-二氟苯甲酰基 硫脲(10): 白色固体, 收率 76\%. m.p. 191 193 ${ }^{\circ} \mathrm{C} ;{ }^{1} \mathrm{H}$ NMR (DMSO- $\left.d_{6}, 300 \mathrm{MHz}\right) \delta$ : $12.58(\mathrm{~s}, 1 \mathrm{H}), 11.48(\mathrm{~s}$, 1H), 10.86 (s, 1H), 7.99 (s, 1H), 7.65 (d, $J=8.3 \mathrm{~Hz}, 2 \mathrm{H})$, 7.51 (t, $J=7.5 \mathrm{~Hz}, 1 \mathrm{H}), 7.24$ (d, $J=8.3 \mathrm{~Hz}, 2 \mathrm{H}), 7.18$ (d, $J=7.5 \mathrm{~Hz}, 2 \mathrm{H}), 4.36(\mathrm{t}, J=4.8 \mathrm{~Hz}, 1 \mathrm{H}), 2.97(\mathrm{~d}, J=4.8$ $\mathrm{Hz}, 2 \mathrm{H})$; IR (KBr) $v$ : 3248, 3056, 1774, 1735, 1672, 1596 $\mathrm{cm}^{-1}$. HR-ESI-MS cacld for $\mathrm{C}_{18} \mathrm{H}_{15} \mathrm{~F}_{2} \mathrm{~N}_{4} \mathrm{O}_{3} \mathrm{~S}[\mathrm{M}+\mathrm{H}]^{+}$ 405.0833, found 405.0836.

5-(4-氨基苯基)-2,4-咪唑啉二酮-2,6-二氟苯甲酰基 硫脲(11): 白色固体, 收率 84\%. m.p. 202 204 ${ }^{\circ} \mathrm{C} ;{ }^{1} \mathrm{H}$ NMR (DMSO- $\left.d_{6}, 300 \mathrm{MHz}\right) \delta$ : $12.60(\mathrm{~s}, 1 \mathrm{H}), 11.52(\mathrm{~s}$, 1H), 10.48 (s, 1H), $8.46(\mathrm{~s}, 1 \mathrm{H}), 7.59$ (d, $J=7.5 \mathrm{~Hz}, 2 \mathrm{H})$, 7.54 (d, $J=7.5 \mathrm{~Hz}, 2 \mathrm{H}), 7.50$ (t, $J=7.4 \mathrm{~Hz}, 1 \mathrm{H}), 7.16$ (d, $J=7.4 \mathrm{~Hz}, 2 \mathrm{H}), 5.22(\mathrm{~s}, 1 \mathrm{H})$; IR (KBr) v: 3242, 3058, 1779, 1725, 1678, $1592 \mathrm{~cm}^{-1}$. HR-ESI-MS cacld for $\mathrm{C}_{17} \mathrm{H}_{13} \mathrm{~F}_{2} \mathrm{~N}_{4} \mathrm{O}_{3} \mathrm{~S}[\mathrm{M}+\mathrm{H}]^{+}$391.0676, found 391.0679.

5-(4-氨基苠基)-2-硫代-2,4-咪唑啉二酮-2,6-二氟苯 甲酰基硫嫝(12): 微黄色固体, 收率 82\%. m.p. 234 236 ${ }^{\circ} \mathrm{C} ;{ }^{1} \mathrm{H}$ NMR (DMSO- $\left.d_{6}, 300 \mathrm{MHz}\right) \delta$ : $12.62(\mathrm{~s}, 1 \mathrm{H}), 11.58$ (s, 1H), 11.52 (s, 1H), 10.09 (s, 1H), 7.66 (d, $J=8.3 \mathrm{~Hz}$, 2H), 7.52 (t, $J=7.5 \mathrm{~Hz}, 1 \mathrm{H}), 7.25$ (d, $J=8.3 \mathrm{~Hz}, 2 \mathrm{H}), 7.17$ (d, $J=7.5 \mathrm{~Hz}, 2 \mathrm{H}), 4.55$ (t, $J=4.8 \mathrm{~Hz}, 1 \mathrm{H}$ ), 2.99 (d, $J=4.8$ $\mathrm{Hz}, 2 \mathrm{H}$ ); IR (KBr) v: 3239, 3061, 1777, 1733, 1679, 1596 $\mathrm{cm}^{-1}$. HR-ESI-MS cacld for $\mathrm{C}_{18} \mathrm{H}_{15} \mathrm{~F}_{2} \mathrm{~N}_{4} \mathrm{O}_{2} \mathrm{~S}_{2}[\mathrm{M}+\mathrm{H}]^{+}$ 421.0604, found 421.0609.

\subsection{2 酰基腿的合成通法}

将 0.88 g 5-(4-氨基苄基)-2-硫代-2,4-咪唑啉二酮(4 $\mathrm{mmol}$ )加入到装有 $30 \mathrm{~mL}$ 二氯乙烷的三口烧瓶中, 溶解 混匀后, 加入新制备的 2,6-二氟苯甲酰异氰酸酯, 补加 $5 \mathrm{~mL}$ DMF, 加热回流过夜. TLC 检测[取样加入少量甲 醇, $V($ 二氯甲烷 $) / V($ 乙醇 $)=20 / 1$, 原料点消失, 停止加
热, 静置冷却, 抽滤, 所得固体经石油醚/乙酸乙酯/ DMF 体系重结晶, 得微黄色固体 $0.52 \mathrm{~g}$, 滤液旋至近 干, 加入 $120 \mathrm{~mL}$ 乙酸乙酯, 用 $90 \mathrm{~mL}$ 饱和食盐水洗 3 次, 无水硫酸钠干燥后旋干, 所得残渣加入石油醚和二 氯甲烷，体系不溶解，再滴加无水甲醇摚拌，析出大量 粉末, 抽滤, 二氯甲烷洗涤固体多次, 干燥, TLC 检测为 目标化合物. 共得 $1.125 \mathrm{~g}$ 黄色固体化合物 $\mathbf{1 5}$, 收率为 70\%. 采用相同方法制得化合物 $\mathbf{1 3}$ 和 14.

5-(4-氨基苄基)-2,4-咪唑啉二酮-2,6-二氟苯甲酰基 嫝(13): 微黄色固体, 收率 $46 \%$. m.p. $236 \sim 238{ }^{\circ} \mathrm{C} ;{ }^{1} \mathrm{H}$ NMR (DMSO- $\left.d_{6}, 300 \mathrm{MHz}\right) \delta$ : $11.39(\mathrm{~s}, 1 \mathrm{H}), 10.44$ (s, $1 \mathrm{H}), 10.13(\mathrm{~s}, 1 \mathrm{H}), 7.93(\mathrm{~s}, 1 \mathrm{H}), 7.68 \sim 7.57(\mathrm{~m}, 1 \mathrm{H}), 7.47$ (d, $J=8.3 \mathrm{~Hz}, 2 \mathrm{H}), 7.28 \sim 7.22(\mathrm{~m}, 2 \mathrm{H}), 7.15(\mathrm{~d}, J=8.3$ $\mathrm{Hz}, 2 \mathrm{H}), 4.31$ (t, $J=4.8 \mathrm{~Hz}, 1 \mathrm{H}), 2.90$ (d, $J=4.8 \mathrm{~Hz}, 2 \mathrm{H}$ ); IR (KBr) v: 3224, 3045, 2934, 1768, 1729, 1691, 1627, 1594, 1545, 1507, 1469, 1419, 1284, 1225, 1013, 917, 798, $748 \mathrm{~cm}^{-1}$. HR-ESI-MS cacld for $\mathrm{C}_{18} \mathrm{H}_{15} \mathrm{~F}_{2} \mathrm{~N}_{4} \mathrm{O}_{4}[\mathrm{M}+\mathrm{H}]^{+}$ 389.1056, found 389.1058; cacld for $\mathrm{C}_{18} \mathrm{H}_{14} \mathrm{~F}_{2} \mathrm{~N}_{4} \mathrm{O}_{4} \mathrm{Na}$ $[\mathrm{M}+\mathrm{Na}]^{+}$411.0881, found 411.0887.

5-(4-氨基苯基)-2,4-咪唑啉二酮-2,6-二氟苯甲酰基 嫝(14): 微黄色固体, 收率 $62 \%$. m.p. 268 $270{ }^{\circ} \mathrm{C} ;{ }^{1} \mathrm{H}$ NMR (DMSO- $\left.d_{6}, 300 \mathrm{MHz}\right) \delta$ : $11.44(\mathrm{~s}, 1 \mathrm{H}), 10.79$ (s, $1 \mathrm{H}), 10.21(\mathrm{~s}, 1 \mathrm{H}), 8.39(\mathrm{~s}, 1 \mathrm{H}), 7.68 \sim 7.58(\mathrm{~m}, 3 \mathrm{H})$, $7.32 \sim 7.23(\mathrm{~m}, 4 \mathrm{H}), 5.15$ (s, 1H); IR (KBr) v: 3196, 3042, 1783, 1729, 1668, 1625, 1605, 1560, 1516, 1465, 1422, 1288, 1232, 1182, 1010, 921, 797, 765, $745 \mathrm{~cm}^{-1}$. HR-ESI-MS cacld for $\mathrm{C}_{17} \mathrm{H}_{13} \mathrm{~F}_{2} \mathrm{~N}_{4} \mathrm{O}_{4}[\mathrm{M}+\mathrm{H}]^{+} 375.0899$, found 375.0900 , cacld for $\mathrm{C}_{17} \mathrm{H}_{12} \mathrm{~F}_{2} \mathrm{~N}_{4} \mathrm{O}_{4} \mathrm{Na}[\mathrm{M}+\mathrm{Na}]^{+}$ 397.0724, found 397.0730.

5-(4-氨基芐基)-2-硫代-2,4-咪唑啉二酮-2,6-二氟苯 甲酰基硫腿(15): 黄色固体，收率 70\%. m.p. 253 255 ${ }^{\circ} \mathrm{C} ;{ }^{1} \mathrm{H}$ NMR (DMSO- $\left.d_{6}, 300 \mathrm{MHz}\right) \delta$ : $11.46(\mathrm{~s}, 1 \mathrm{H}), 11.40$ (s, 1H), 10.14 (s, 1H), 10.08 (s, 1H), 7.65 7.57 (m, 1H), $7.46(\mathrm{~d}, J=8.3 \mathrm{~Hz}, 2 \mathrm{H}), 7.28 \sim 7.22(\mathrm{~m}, 2 \mathrm{H}), 7.14(\mathrm{~d}, J=$ $8.3 \mathrm{~Hz}, 2 \mathrm{H}), 4.55(\mathrm{t}, J=4.8 \mathrm{~Hz}, 1 \mathrm{H}), 2.96(\mathrm{~d}, J=4.8 \mathrm{~Hz}$, 2H); IR (KBr) v: 3196, 3121, 3050, 2919, 1739, 1694, 1624, 1606, 1561, 1535, 1491, 1469, 1419, 1285, 1232, $1172,1017,919,804,776 \mathrm{~cm}^{-1}$. HR-ESI-MS cacld for $\mathrm{C}_{18} \mathrm{H}_{15} \mathrm{~F}_{2} \mathrm{~N}_{4} \mathrm{O}_{3} \mathrm{~S}[\mathrm{M}+\mathrm{H}]^{+}$405.0827, found 405.0827; cacld for $\mathrm{C}_{18} \mathrm{H}_{14} \mathrm{~F}_{2} \mathrm{~N}_{4} \mathrm{O}_{3} \mathrm{SNa}[\mathrm{M}+\mathrm{Na}]^{+} 427.0652$, found 427.0653 .

\section{References}

[1] Saeed, S.; Florke, U.; Erben, M. F. J. Sulfur Chem. 2014, 35, 318.

[2] Zhang, J. F.; Liu, C.; Ma, Y.; Wang, B. L.; Xiong, L. X.; Yu, S. J.; Li, Z. M. Lett. Drug Des. Discovery 2013, 10, 497. 
[3] Zhang, J. F.; Xu, J. Y.; Wang, B. L.; Li, Y. X.; Xiong, L. X.; Li, Y. Q.; Ma, Y.; Li, Z. M. J. Agric. Food Chem. 2012, 60, 7565.

[4] Sun, R. F.; Lv, M. Y.; Chen, L.; Li, Q. S.; Song, H. B.; Huang, R. Q.; Bi, F. C.; Wang, Q. M. J. Agric. Food Chem. 2008, 56, 11376.

[5] Shang, J.; Sun, R. F.; Li, Y. Q.; Huang, R. Q.; Bi, F. C.; Wang, Q. M. J. Agric. Food Chem. 2010, 58, 1834.

[6] Sun, Z. H.; Huang, W.; Gong, Y. Y.; Lan, J.; Liu, X. H.; Weng, J. Q.; Li, Y. S.; Tan, C. X. Chin. J. Org. Chem. 2013, 33, 2612 (in Chinese).

(孙召慧, 黄伟, 贡云芸, 蓝健, 刘幸海, 翁建全, 李永曙, 谭成 侠, 有机化学, 2013, 33, 2612.)

[7] Gong, Y. Y.; Huang, W.; Liu, X. H.; Weng, J. Q.; Tan, C. X. Chin. J. Org. Chem. 2013, 33, 2396 (in Chinese).

(贡云芸, 黄伟, 刘幸海, 翁建全, 谭成侠, 有机化学, 2013, 33, 2396.)

[8] Dai, H.; Zhao, Y. H.; Niu, P.; Qian, Y. J.; Li, Y. Q.; Fang, J. X.; Shi, Y. J. Chin. J. Org. Chem. 2013, 33, 1568 (in Chinese).

(戴红, 赵元飞, 牛平, 钱翊钧, 李永强, 方建新, 石玉军, 有机 化学, 2013, 33, 1568.)

[9] Han, J.; Wang, J.; Dong, H.; Xu, Z. H.; Liu, B.; Wang, M. A. Chin.
J. Org. Chem. 2013, 33, 596 (in Chinese).

(韩金涛，王进敏，董宏波，徐志红，刘斌，王明安，有机化学， 2013, 33, 596.)

[10] Wang, J. M.; Xu, Z. H.; Han, J. T.; Dong, H. B.; Liu, B.; Wang, M. A. Chin. J. Org. Chem. 2013, 33, 2186 (in Chinese). (王进敏, 徐志红, 韩金涛, 董宏波, 刘斌, 王明安, 有机化学, 2013, 33, 2186.)

[11] Han, J. T.; Dong, H. B.; Xu, Z. H.; Wang, J. M.; Wang, M. A. Int. J. Mol. Sci. 2013, 14, 19526.

[12] Bjerglund, K.; Lindhardt, A. T.; Skrydstrup, T. J. Org. Chem. 2012, $77,3793$.

[13] Liptrot, D.; Alcaraz, L.; Roberts, B. Adv. Synth. Catal. 2010, 352, 2183.

[14] Seiller, B.; Heins, D.; Bruneau, C.; Dixneuf, P. H. Tetrahedron 1995, 51, 10901 .

[15] Sun, R. F.; Zhang, Y. L.; Bi, F. C.; Wang, Q. M. J. Agric. Food Chem. 2009, 57, 6356.

[16] Sun, R. F. Ph.D Dissertation, Nankai University, Tianjin, 2010 (in Chinese).

(孙然锋, 博士学位论文, 南开大学, 天津, 2010.) 